\title{
Pleural effusion as a novel prognostic factor in metastatic thyroid carcinoma
}

\author{
David T Broome ${ }^{11}$, Gauri B Gadre ${ }^{2}$, Ehsan Fayazzadeh ${ }^{3}$, James F Bena ${ }^{4}$ and Christian Nasr ${ }^{1}$ \\ 'Department of Endocrinology, Diabetes \& Metabolism, Cleveland Clinic Foundation, Cleveland, Ohio, USA \\ 2Department of Internal Medicine, Scripps Clinic, La Jolla, California, USA \\ ${ }^{3}$ Department of Radiology, Cleveland Clinic Foundation, Cleveland, Ohio, USA \\ ${ }^{4}$ Department of Quantitative Health Sciences, Cleveland Clinic Foundation, Cleveland, Ohio, USA \\ Correspondence should be addressed to C Nasr: nasrc@ccf.org
}

\begin{abstract}
Objective: To identify novel prognostic risk factors and compare them with other known prognostic risk factors in follicular-cell-derived thyroid carcinoma (FDTC) with distant metastases.

Methods: A retrospective review was conducted of adult patients with metastatic FDTC seen at a tertiary care center between January 1990 and December 2010. A 15-year Kaplan-Meier survival estimate was created for overall survival (OS) and cancer-specific survival (CSS). Hazard ratios (HR) and $P$ values from Cox proportional hazard models were used with a $95 \% \mathrm{Cl}$.

Results: There were 143 patients (60.1\% male, 39.9\% female), of whom 104 (72.7\%) patients had papillary, 30 (21.0\%) had follicular, 5 (3.5\%) had poorly differentiated, and 4 (2.8\%) had Hürthle cell cancers. Median length of follow-up was 80.0 months (range 1.0-564.0). The 15-year mortality rate was $32.2 \%$ and cancer-specific mortality was $25.2 \%$, with OS and CSS having the same risk factors. Lung was the most common site of metastases in 53 patients (37.1\%), and patients with pleural effusions had significantly lower CSS (HR = 5.21, CI=1.79-15.12). Additional risk factors for a decreased CSS included: older age upon diagnosis ( $>45$ years, $\mathrm{HR}=4.15, \mathrm{Cl}=1.43-12.02$ ), multiple metastatic locations ( $\mathrm{HR}=3.75, \mathrm{Cl}=1.32-10.67)$, and incomplete/unknown tumor resection ( $\mathrm{HR}=2.35, \mathrm{Cl}=1.18-4.67)$.

Conclusion: This study is the first to demonstrate that pleural effusion is a poor prognostic sign in patients with FDTC with distant metastases and compare this risk with other accepted prognostic variables.
\end{abstract}

\section{Introduction}

Thyroid cancer is currently the third fastest rising cancer diagnosis in the United States. The National Cancer Institute indicates that there were 52,070 new cases of thyroid cancer diagnosed in 2019 and an estimated 52,890 newly diagnosed cases in 2020, representing $2.9 \%$ of all new cancer diagnoses in the United States (1). The incidence of thyroid carcinoma is 15.7 per 100,000 men and women per year and represents $0.4 \%$ of all cancer deaths currently. The incidence has increased significantly since 1975 going from 4.5 per 100,000 in 1975 to 15.7 per 100,000 in 2020, representing a 3.4-fold increase (1). The rise in incidence is mainly attributed to an increase in thyroid cancer detection secondary to increased imaging, increased detection of incidental thyroid tumors, and introduction of high-sensitivity ultrasonography detecting small subcentimeter lesions. Malignant pleural effusion currently has an estimated annual incidence of 150,000 in the USA, and it is expected that with the https://ec.bioscientifica.com

https://doi.org/10.1530/EC-20-0193 (c) 2020 The authors Published by Bioscientifica Ltd

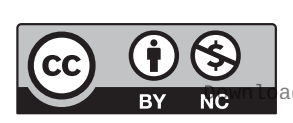

This work is licensed under a Creative Commons Attribution-NonCommercial 4.0 International License. ded from Bioscientifica.com at 04/26/2023 11:23:16AM 
increase in new cancer diagnoses, this incidence would also increase $(2,3,4)$.

Survival for low-risk thyroid cancer remains above 90\% (1), and prognosis for patients with metastasis on presentation falls between 23.1 and $31 \%$ for 10-year survival $(5,6,7)$. The majority of cases are low grade/ stage upon diagnosis and seldom do patients present with distant metastasis, which have since made it difficult to assess which clinical characteristics have the largest effect on overall survival (OS) and cancer-specific survival (CSS). Significant risk factors that were identified for a decrease in survival included follicular thyroid cancer, age $>45$ years (8), cancer with metastasis to bone (5), increased tumor size (8) and tumor size $>3 \mathrm{~cm}$ (5), and incomplete tumor resection $(5,8)$. In addition to extra-thyroid tumor spread, lymph node metastases, distant metastases and tall cell variants for PTC (8), other risk factors associated with a decrease in CSS were distant metastasis diagnosed before whole body scan, non-radioactive iodine (RAI) avidity, external beam radiation to distant metastases $(6,7)$, and a higher MACIS score (9). Treatment with RAI was previously found to be the single most powerful prognostic indicator for increased disease-free interval and significantly increased survival, while patients derived no benefit from treatment with external radiation (6). It is important to note that pleural effusion has not been studied with respect to these variables and its relative importance has never been quantified with respect to known prognostic variables in metastatic thyroid carcinoma.

The aim of this study was to identify the effect of novel prognostic variables (such as pleural effusion) on OS and CSS in comparison to known prognostic variables in patients with follicular-cell-derived thyroid carcinoma (FDTC) with distant metastases.

\section{Methods}

\section{Patients}

This study was approved by the Cleveland Clinic Foundation Institutional Review Board/Independent Ethics Committee (IRB/IEC) and was a retrospective review of patients (18 years and older) who presented to a tertiary referral center with FDTC between January 1, 1990 and December 31, 2010. Consent was determined to not be necessary and was waived given the minimal risk posed to patients for this study. Patients were identified with a diagnosis of thyroid cancer utilizing ICD-9 codes, then individual chart review was performed to ensure inclusion and exclusion criteria were met. Inclusion criteria included thyroid carcinoma with distant metastases and age greater than 18 years or older on presentation to the tertiary referral center. Patients excluded from analysis were those not followed at the tertiary referral center, those with insufficient data, and patients who had other types of concomitant cancer (e.g. breast, colon cancer). Additionally, medullary thyroid carcinoma patients were excluded. After manual chart review for exclusion criteria, 143 patients were included in the analysis (Supplementary Fig. 1, see section on supplementary materials given at the end of this article).

\section{Data collection process}

Data were confirmed/refuted from the electronic research informatics with manual chart review for inclusion/ exclusion criteria. Disputed results were discussed and agreed upon by all data collectors (D T B and G B G) and investigators $(\mathrm{C} \mathrm{N})$. The following demographic factors were collected: age, age $\geq 45$ years, last recorded age, gender, race, history of neck radiation exposure, history of preexisting thyroid disease, last vital status, length of follow-up (months), and death due to disease. Death due to disease was defined as dying from the primary thyroid cancer or a direct complication of thyroid cancer, including expiring in hospice (if was transitioned to hospice for management of thyroid cancer), hypoxia from malignant pleural effusion, hypoxia from respiratory failure if determined to be from disease burden.

\section{Statistical analysis}

Categorical variables were summarized using frequencies and percentages, while continuous measures were reported with medians and ranges. Two separate univariate analyses were performed with primary outcomes of overall survival and cause-specific survival. The number of deaths and deaths due to cancer were summarized with frequencies and percentages, while 15-year Kaplan-Meier survival estimates were created for overall and causespecific survival. Hazard ratios (HR) and $P$ values from Cox proportional hazard models were used with 95\% CI. A $P$ value less than 0.05 was set as statistically significant. Multivariable models were fit using stepwise selection, with significance criteria of 0.05 to enter and remain in the model. Analysis was performed with SAS software (version 9.4; Cary, NC, USA).
This work is licensed under a Creative Commons Attribution-NonCommercial 4.0 International License. ded from Bioscientifica.com at 04/26/2023 11:23:16AM via free access 


\section{Results}

\section{Patient demographics}

There were 143 patients who met study inclusion criteria after applying exclusion criteria (Supplementary Fig. 1). The median age at diagnosis was 59.0 years $(9.0,89.0)$, with patients who developed lung metastases and a pleural effusion being older at a median age of $75.0(28.0,89.0)$. The majority were male $(60.1 \%)$ patients and the most common races represented were White $(83.9 \%, n=120)$ and Black or African American $(10.5 \%, n=15)$. The mean length of follow-up was 80.0 months $(1.00,564.0)$, with $32.2 \%$ being dead after 15 years of follow-up $(n=46)$, with thyroid cancer being the most common cause of death $(n=36)$. Patients who developed a pleural effusion had a median length of follow-up of 15.0 months $(1.00,432.00)$ (Table 1). Comparisons between the total cohort, patients with lung metastases with and without pleural effusion were compared and included in Tables 1 and 2 .

\section{Disease characteristics}

The most common cancer type was papillary thyroid cancer $(72.7 \%, n=104)$, with the most common histologic subtypes listed in Table 2 . The median size of the tumor was $3.6 \mathrm{~cm}(0.2,14.0)$, and $78.3 \%$ had complete resection of their tumor $(n=112)$. The majority of patients did not have visible lung metastases on diagnosis $(62.9 \%, n=90)$, but $37.1 \%$ of patients had developed lung metastases $(n=53)$ during follow-up (Table 2$)$. In the patients with lung

Table 1 Demographics summaries.

\begin{tabular}{|c|c|c|c|c|c|c|c|}
\hline \multirow[b]{2}{*}{ Factor } & \multirow[b]{2}{*}{$\begin{array}{c}\text { Total } \\
(n=143)\end{array}$} & \multicolumn{2}{|c|}{ Lung metastasis } & \multirow[b]{2}{*}{ P-value } & \multicolumn{2}{|c|}{$\begin{array}{l}\text { Lung metastasis with } \\
\text { pleural effusion }\end{array}$} & \multirow[b]{2}{*}{ P-value } \\
\hline & & $\begin{array}{c}\text { No } \\
(n=90)\end{array}$ & $\begin{array}{c}\text { Yes } \\
(n=53)\end{array}$ & & $\begin{array}{c}\text { No } \\
(n=43)\end{array}$ & $\begin{array}{c}\text { Yes } \\
(n=10)\end{array}$ & \\
\hline Age at diagnosis & $\begin{array}{c}59.0 \\
(9.0,89.0)\end{array}$ & $\begin{array}{c}58.0 \\
(9.0,81.0)\end{array}$ & $\begin{array}{c}60.0 \\
(13.0,89.0)\end{array}$ & $0.11^{b}$ & $\begin{array}{c}58.0 \\
(13.0,85.0)\end{array}$ & $\begin{array}{c}75.0 \\
(28.0,89.0)\end{array}$ & $0.020^{b}$ \\
\hline Age $>$ or $=45$ & $107(74.8)$ & $67(74.4)$ & $40(75.5)$ & $0.89^{c}$ & $31(72.1)$ & $9(90.0)$ & $0.24^{c}$ \\
\hline Last Age & $\begin{array}{c}64.0 \\
(24.0,91.0)\end{array}$ & $\begin{array}{c}65.0 \\
(24.0,91.0)\end{array}$ & $\begin{array}{c}62.0 \\
(26.0,89.0)\end{array}$ & $0.76^{b}$ & $\begin{array}{c}60.0 \\
(26.0,87.0)\end{array}$ & $\begin{array}{c}78.0 \\
(58.0,89.0)\end{array}$ & $0.010^{b}$ \\
\hline Gender & & & & $0.96^{c}$ & & & $0.49^{c}$ \\
\hline Female & 57 (39.9) & $36(40.0)$ & $21(39.6)$ & & $18(41.9)$ & $3(30.0)$ & \\
\hline Male & $86(60.1)$ & $54(60.0)$ & $32(60.4)$ & & $25(58.1)$ & $7(70.0)$ & \\
\hline Race & & & & $0.25^{d}$ & & & $0.58^{d}$ \\
\hline Pacific Islander & $1(0.70)$ & $1(1.1)$ & $0(0.0)$ & & $0(0.0)$ & $0(0.0)$ & \\
\hline Black or African American & $15(10.5)$ & $6(6.7)$ & $9(17.0)$ & & $6(14.0)$ & $3(30.0)$ & \\
\hline White & $120(83.9)$ & $78(86.7)$ & $42(79.2)$ & & $35(81.4)$ & $7(70.0)$ & \\
\hline More than one race & $2(1.4)$ & $2(2.2)$ & $0(0.0)$ & & $0(0.0)$ & $0(0.0)$ & \\
\hline Unknown/not reported & $5(3.5)$ & $3(3.3)$ & $2(3.8)$ & & $2(4.7)$ & $0(0.0)$ & \\
\hline $\begin{array}{l}\text { History of neck radiation } \\
\text { exposure }\end{array}$ & & & & $0.84^{c}$ & & & $0.99^{d}$ \\
\hline No & $133(93.0)$ & 84 (93.3) & $49(92.5)$ & & $40(93.0)$ & $9(90.0)$ & \\
\hline Yes & $10(7.0)$ & $6(6.7)$ & $4(7.5)$ & & $3(7.0)$ & $1(10.0)$ & \\
\hline $\begin{array}{l}\text { History of preexisting } \\
\text { thyroid disease }\end{array}$ & & & & $0.10^{d}$ & & & $0.42^{c}$ \\
\hline None & $121(84.6)$ & $79(87.8)$ & $42(79.2)$ & & $42(79.2)$ & $35(81.4)$ & \\
\hline Graves' disease & $2(1.4)$ & $2(2.2)$ & $0(0.0)$ & & $0(0.0)$ & $0(0.0)$ & \\
\hline Hashimoto's thyroiditis & $1(0.70)$ & $1(1.1)$ & $0(0.0)$ & & $0(0.0)$ & $0(0.0)$ & \\
\hline Others & $19(13.3)$ & $8(8.9)$ & $11(20.8)$ & & $11(20.8)$ & $8(18.6)$ & \\
\hline Last vital status & & & & $0.70^{c}$ & & & $0.002^{c}$ \\
\hline Alive & $97(67.8)$ & $60(66.7)$ & $37(69.8)$ & & $34(79.1)$ & $3(30.0)$ & \\
\hline Dead & $46(32.2)$ & $30(33.3)$ & $16(30.2)$ & & $9(20.9)$ & $7(70.0)$ & \\
\hline $\begin{array}{l}\text { Length of follow-up } \\
\text { (months) }\end{array}$ & $\begin{array}{c}80.0 \\
(1.00,564.0)\end{array}$ & $\begin{array}{c}99.5 \\
(3.0,564.0)\end{array}$ & $\begin{array}{c}56.0 \\
(1.00,432.0)\end{array}$ & $<0.001^{b}$ & $\begin{array}{c}64.0 \\
(3.0,210.0)\end{array}$ & $\begin{array}{c}15.0 \\
(1.00,432.0)\end{array}$ & $0.015^{b}$ \\
\hline Death due to disease & & & & $0.11^{d}$ & & & $0.44^{d}$ \\
\hline Yes & $36(78.3)$ & $21(70.0)$ & $15(93.8)$ & & $9(100.0)$ & $6(85.7)$ & \\
\hline No & $4(8.7)$ & $3(10.0)$ & $1(6.3)$ & & $0(0.0)$ & 1 (14.3) & \\
\hline Unknown & $6(13.0)$ & $6(20.0)$ & $0(0.0)$ & & $0(0.0)$ & $0(0.0)$ & \\
\hline
\end{tabular}

Statistics presented as Median (min, $\max$ ) or $n$ (column \%).

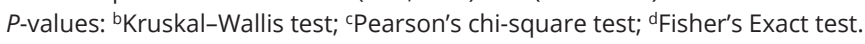

https://ec.bioscientifica.com

https://doi.org/10.1530/EC-20-0193 (c) 2020 The authors Published by Bioscientifica Ltd

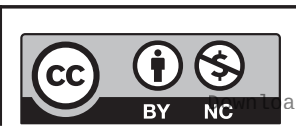

This work is licensed under a Creative Commons Attribution-NonCommercial 4.0 International License. ded from Bioscientifica.com at 04/26/2023 11:23:16AM via free access 
Table 2 Thyroid summaries.

\begin{tabular}{|c|c|c|c|c|c|c|c|}
\hline \multirow[b]{2}{*}{ Factor } & \multirow[b]{2}{*}{$\begin{array}{c}\text { Total } \\
(n=143) \\
\end{array}$} & \multicolumn{2}{|c|}{ Lung metastasis } & \multirow[b]{2}{*}{ P-value } & \multicolumn{2}{|c|}{$\begin{array}{c}\text { Lung metastasis with pleural } \\
\text { effusion }\end{array}$} & \multirow[b]{2}{*}{$P$-value } \\
\hline & & $\begin{array}{c}\text { No } \\
(n=90) \\
\end{array}$ & $\begin{array}{c}\text { Yes } \\
(n=53) \\
\end{array}$ & & $\begin{array}{c}\text { No } \\
(n=43)\end{array}$ & $\begin{array}{c}\text { Yes } \\
(n=10) \\
\end{array}$ & \\
\hline Cancer type & & & & $0.21^{d}$ & & & $0.089^{d}$ \\
\hline Papillary thyroid cancer & $104(72.7)$ & $62(68.9)$ & $42(79.2)$ & & $36(83.7)$ & $6(60.0)$ & \\
\hline Follicular thyroid cancer & $30(21.0)$ & $23(25.6)$ & $7(13.2)$ & & $5(11.6)$ & $2(20.0)$ & \\
\hline Hürthle cell cancer & $4(2.8)$ & $3(3.3)$ & $1(1.9)$ & & $1(2.3)$ & $0(0.0)$ & \\
\hline $\begin{array}{l}\text { Poorly differentiated } \\
\text { cancer }\end{array}$ & $5(3.5)$ & $2(2.2)$ & $3(5.7)$ & & $1(2.3)$ & $2(20.0)$ & \\
\hline $\begin{array}{l}\text { Histologic subtype: tall cell } \\
\text { variant }\end{array}$ & $20(14.0)$ & $7(7.8)$ & $13(24.5)$ & $0.005^{c}$ & $12(27.9)$ & $1(10.0)$ & $0.24^{c}$ \\
\hline $\begin{array}{l}\text { Histologic subtype: follicular } \\
\text { variant }\end{array}$ & $32(22.4)$ & $15(16.7)$ & $17(32.1)$ & $0.033^{c}$ & $14(32.6)$ & $3(30.0)$ & $0.88^{c}$ \\
\hline $\begin{array}{l}\text { Histologic subtype: diffuse } \\
\text { sclerosing variant }\end{array}$ & $4(2.8)$ & $3(3.3)$ & $1(1.9)$ & $0.99^{d}$ & $1(2.3)$ & $0(0.0)$ & $0.99^{d}$ \\
\hline Histologic subtype: oncocytic & $6(4.2)$ & $4(4.4)$ & $2(3.8)$ & $0.99^{d}$ & $1(2.3)$ & $1(10.0)$ & $0.34^{d}$ \\
\hline $\begin{array}{l}\text { Histologic subtype: } \\
\text { cribriform morular }\end{array}$ & $0(0.0)$ & $0(0.0)$ & $0(0.0)$ & & $0(0.0)$ & $0(0.0)$ & \\
\hline $\begin{array}{l}\text { Histologic subtype: solid } \\
\text { variant }\end{array}$ & $3(2.1)$ & $1(1.1)$ & $2(3.8)$ & $0.56^{d}$ & $1(2.3)$ & $1(10.0)$ & $0.34^{d}$ \\
\hline $\begin{array}{l}\text { Histologic subtype: } \\
\text { micropapillary (Hobnail) }\end{array}$ & $2(1.4)$ & $0(0.0)$ & $2(3.8)$ & $0.14^{d}$ & $2(4.7)$ & $0(0.0)$ & $0.99^{d}$ \\
\hline $\begin{array}{l}\text { Histologic subtype: } \\
\text { encapsulated papillary }\end{array}$ & $0(0.0)$ & $0(0.0)$ & $0(0.0)$ & & $0(0.0)$ & $0(0.0)$ & \\
\hline Histologic subtype: insular & $8(5.6)$ & $4(4.4)$ & $4(7.5)$ & $0.44^{c}$ & $2(4.7)$ & $2(20.0)$ & $0.16^{d}$ \\
\hline $\begin{array}{l}\text { Histologic subtype: Warthin } \\
\text { like }\end{array}$ & $0(0.0)$ & $0(0.0)$ & $0(0.0)$ & & $0(0.0)$ & $0(0.0)$ & \\
\hline $\begin{array}{l}\text { Histologic subtype: Not } \\
\text { stated }\end{array}$ & $76(53.1)$ & $55(61.1)$ & $21(39.6)$ & $0.013^{c}$ & 15 (34.9) & $6(60.0)$ & $0.14^{c}$ \\
\hline Largest tumor size known & $97(67.8)$ & $56(62.2)$ & $41(77.4)$ & $0.061^{c}$ & $36(83.7)$ & $5(50.0)$ & $0.022^{c}$ \\
\hline Tumor size & $\begin{array}{c}3.6 \\
(0.20,14.0)\end{array}$ & $\begin{array}{c}4.0 \\
(0.20,12.0)\end{array}$ & $\begin{array}{c}3.0 \\
(1.00,14.0)\end{array}$ & $0.74^{\mathrm{b}}$ & $\begin{array}{c}3.0 \\
(1.5,14.0)\end{array}$ & $\begin{array}{c}7.0 \\
(1.00,9.0)\end{array}$ & $0.60^{\mathrm{b}}$ \\
\hline Completeness of surgery & & & & $0.53^{c}$ & & & $0.21^{\mathrm{c}}$ \\
\hline Completely resected & $112(78.3)$ & $72(80.0)$ & $40(75.5)$ & & $34(79.1)$ & $6(60.0)$ & \\
\hline Incompletely resected & $31(21.7)$ & $18(20.0)$ & $13(24.5)$ & & 9 (20.9) & $4(40.0)$ & \\
\hline $\begin{array}{l}\text { Lung metastases: } \\
\text { micronodules (up to } 9 \text { mm) }\end{array}$ & $45(31.5)$ & --- & $45(84.9)$ & & $37(86.0)$ & $8(80.0)$ & $0.63^{c}$ \\
\hline $\begin{array}{l}\text { Lung metastases: } \\
\text { macronodules }(>1 \mathrm{~cm})\end{array}$ & $19(13.3)$ & --- & $19(35.8)$ & & $13(30.2)$ & $6(60.0)$ & $0.077^{c}$ \\
\hline $\begin{array}{l}\text { Lung metastases: diffuse } \\
\text { miliary }\end{array}$ & $2(1.4)$ & ---- & $2(3.8)$ & & $2(4.7)$ & $0(0.0)$ & $0.99^{d}$ \\
\hline Number of micronodules & & & & & & & $0.10^{d}$ \\
\hline 0 & $3(6.7)$ & ---- & $3(6.7)$ & & $1(2.7)$ & $2(25.0)$ & \\
\hline 1-5 nodules & $16(35.6)$ & ---- & $16(35.6)$ & & $15(40.5)$ & $1(12.5)$ & \\
\hline 6-10 nodules & $6(13.3)$ & ---- & $6(13.3)$ & & $5(13.5)$ & $1(12.5)$ & \\
\hline$>10$ nodules & $20(44.4)$ & ---- & $20(44.4)$ & & $16(43.2)$ & $4(50.0)$ & \\
\hline Number of macronodules & & & & & & & $0.74^{d}$ \\
\hline 0 & $1(5.3)$ & ---- & $1(5.3)$ & & $0(0.0)$ & $1(16.7)$ & \\
\hline $1-5$ nodules & $11(57.9)$ & ---- & $11(57.9)$ & & $8(61.5)$ & $3(50.0)$ & \\
\hline $6-10$ nodules & $4(21.1)$ & --- & $4(21.1)$ & & $3(23.1)$ & $1(16.7)$ & \\
\hline$>10$ nodules & $3(15.8)$ & ---- & $3(15.8)$ & & $2(15.4)$ & $1(16.7)$ & \\
\hline Laterality in lungs & & & & & & & $0.29^{c}$ \\
\hline Unilateral & $12(22.6)$ & ---- & $12(22.6)$ & & $11(25.6)$ & $1(10.0)$ & \\
\hline Bilateral & 41 (77.4) & ---- & $41(77.4)$ & & $32(74.4)$ & $9(90.0)$ & \\
\hline $\begin{array}{l}\text { Mostly central lung } \\
\text { metastases }\end{array}$ & $5(3.5)$ & ---- & $5(9.4)$ & & 5 (11.6) & $0(0.0)$ & $0.57^{d}$ \\
\hline $\begin{array}{l}\text { Mostly peripheral lung } \\
\text { metastases }\end{array}$ & $44(30.8)$ & ---- & $44(83.0)$ & & $36(83.7)$ & $8(80.0)$ & $0.78^{c}$ \\
\hline
\end{tabular}

https://ec.bioscientifica.com

https://doi.org/10.1530/EC-20-0193 (c) 2020 The authors Published by Bioscientifica Ltd

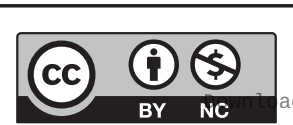

This work is licensed under a Creative Commons Attribution-NonCommercial 4.0 International License. 
Table 2 Continued.

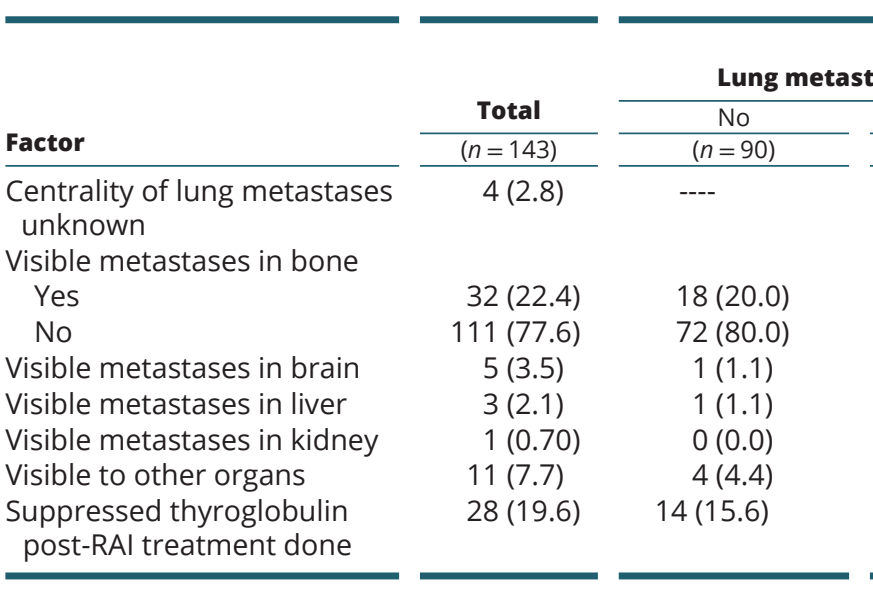

Statistics presented as Median (min, max) or $n$ (column \%).

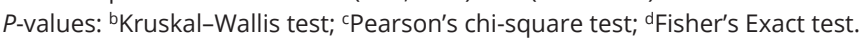

metastases, a pleural effusion developed in ten patients, with eight being bilateral and two being unilateral (Table 2 ). The median MACIS score (9) upon diagnosis was 8.7 $(4.5,12.1)$, and all patients had AJCC seventh edition stage IVc disease (10) and were ATA high risk (11) (Table 3).

\section{Risk factors for overall and cancer-specific survival}

Significant risk factors were the same for OS and CSS with similar hazard ratios. Significant risk factors for decreased CSS included age $\geq 45$ years ( $\mathrm{HR} 4.15$, $\mathrm{CI}=1.43-12.02$,

Table 3 Staging summaries.

\begin{tabular}{|c|c|}
\hline & Total \\
\hline Factor & $(n=143)$ \\
\hline MACIS score & $8.7(4.5,12.1)$ \\
\hline \multicolumn{2}{|l|}{$\mathrm{T}$} \\
\hline$x$ & $33(23.1 \%)$ \\
\hline $1 \mathrm{a}$ & $2(1.4 \%)$ \\
\hline $1 b$ & $2(1.4 \%)$ \\
\hline 2 & $12(8.4 \%)$ \\
\hline 3 & $44(30.8 \%)$ \\
\hline $4 a$ & $45(31.5 \%)$ \\
\hline $4 b$ & $5(3.5 \%)$ \\
\hline \multicolumn{2}{|l|}{$n$} \\
\hline 0 & $28(19.6 \%)$ \\
\hline $1 a$ & $10(7.0 \%)$ \\
\hline $1 b$ & $78(54.5 \%)$ \\
\hline$x$ & 27 (18.9\%) \\
\hline \multicolumn{2}{|l|}{ M } \\
\hline M1 & $143(100.0 \%)$ \\
\hline \multicolumn{2}{|l|}{ TNM stage } \\
\hline IVc & $143(100.0 \%)$ \\
\hline \multicolumn{2}{|c|}{ ATA risk stratification } \\
\hline High & $143(100.0 \%)$ \\
\hline
\end{tabular}

Statistics presented as Median (min, max) or $n$ (column \%).
$P=0.009$ ), multisite metastases (HR 3.75, CI =1.32-10.67, $P=0.013$ ), incomplete tumor resection (HR 2.35, $\mathrm{CI}=1.18-4.67, P=0.014)$, visible metastases in bone (HR $3.20, \mathrm{CI}=1.49-6.88, P=0.003)$, visible metastases in other organs (HR 2.27, $\mathrm{CI}=1.12-4.60, P=0.022$ ), higher age at diagnosis (HR 1.06, $\mathrm{CI}=1.03-1.08, P<0.001$ ), and higher MACIS score (HR 1.98, CI $=1.49-2.63, P<0.001$ ) (Tables 4,5 and 6 ). The presence of visible lung metastases at diagnosis was found to have a decreased risk of mortality when compared to other sites of metastases (HR 0.49, $\mathrm{CI}=0.25-0.97, P=0.042$ ) (Tables 4, 5 and 6). However, in patients who developed lung metastases and a pleural effusion, there was a significant decrease in OS (HR 5.74, $\mathrm{CI}=1.97-16.73, P=0.001$ ) and CSS (HR 4.93, CI= 1.59-15.26, $P=0.006$ ) (Tables 4, 5 and 6). Kaplan-Meier curves for 15-year OS and CSS, for OS and CSS by site of metastases, and the presence of pleural effusion are shown in Figs 1, 2 and 3.

\section{Multivariable analysis}

Significant risk factors identified for decreased CSS included lung metastases with pleural effusion (HR 5.21, $\mathrm{CI}=1.79-15.12, P=0.001)$ and an increased MACIS score (HR 1.86, CI=1.38-2.49, $P<0.001$ ). A significant risk factor that was associated with an increase in CSS was radioactive iodine treatment (HR 0.23, CI=0.09-0.65, $P=0.005$ ) (Table 7). The median RAI treatment dose was $248 \mathrm{mCi}$ (IQ1 112, IQ3 356.9, IQR of 244.9). Although, this association was not further explored, it is thought that patients with RAI-avid disease are more likely to have a better prognosis than those with RAI refractory disease as previously supported in the literature (7).

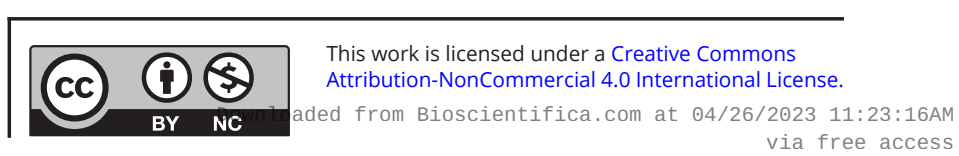


Table 4 Univariate overall survival summary.

\begin{tabular}{|c|c|c|c|c|c|c|}
\hline Variable & $n$ & Events & $\begin{array}{c}\text { 15-year survival } \\
\%(95 \% \mathrm{Cl})\end{array}$ & $\begin{array}{l}\text { Cox univariate hazard } \\
\text { ratio }(95 \% \mathrm{Cl})\end{array}$ & $\begin{array}{l}\text { Cox univariate } \\
\text { wald } P \text {-value }\end{array}$ & $\begin{array}{l}\text { Cox univariate } \\
\text { overall P-value }\end{array}$ \\
\hline Age $>$ or $=45$ & & & & & & 0.003 \\
\hline No & 36 & $7(19 \%)$ & $89.6(78.2,100.0)$ & 1.00 (REF) & & \\
\hline Yes & 107 & $39(36 \%)$ & $47.5(33.0,62.0)$ & $4.15(1.61,10.70)$ & 0.003 & \\
\hline Papillary cancer & & & & & & 0.14 \\
\hline Papillary thyroid cancer & 104 & $30(29 \%)$ & $65.9(54.0,77.8)$ & 1.00 (REF) & & \\
\hline Other thyroid cancer & 39 & $16(41 \%)$ & $44.8(22.1,67.5)$ & $1.59(0.86,2.95)$ & 0.14 & \\
\hline Metastasis location & & & & & & 0.015 \\
\hline Lung only & 45 & $6(13 \%)$ & $82.2(67.3,97.0)$ & 1.00 (REF) & & \\
\hline Bone only & 12 & $1(8 \%)$ & $90.9(73.9,100.0)$ & $0.65(0.08,5.42)$ & 0.69 & \\
\hline Other/multisite & 86 & $39(45 \%)$ & $49.4(36.3,62.6)$ & $3.08(1.30,7.30)$ & 0.011 & \\
\hline Multiple locations & & & & & & 0.003 \\
\hline No & 58 & $7(12 \%)$ & $83.2(70.1,96.3)$ & 1.00 (REF) & & \\
\hline Yes & 85 & $39(46 \%)$ & $49.1(36.0,62.2)$ & $3.42(1.52,7.67)$ & 0.003 & \\
\hline Completeness of surgery & & & & & & 0.005 \\
\hline Tumor completely resected & 112 & $27(24 \%)$ & $67.0(54.9,79.2)$ & 1.00 (REF) & & \\
\hline Tumor incompletely resected & 31 & $19(61 \%)$ & $39.4(19.2,59.7)$ & $2.40(1.31,4.39)$ & 0.005 & \\
\hline Visible metastasis to lung & & & & & & 0.84 \\
\hline No & 22 & $6(27 \%)$ & $74.6(55.2,94.1)$ & 1.00 (REF) & & \\
\hline Yes & 121 & $40(33 \%)$ & $58.1(46.1,70.1)$ & $1.09(0.46,2.60)$ & 0.84 & \\
\hline Visible metastasis to bone & & & & & & 0.007 \\
\hline No & 74 & $14(19 \%)$ & $76.1(64.0,88.2)$ & 1.00 (REF) & & \\
\hline Yes & 69 & $32(46 \%)$ & $45.1(29.5,60.6)$ & $2.42(1.28,4.57)$ & 0.007 & \\
\hline $\begin{array}{l}\text { Visible metastasis in other } \\
\text { organs }\end{array}$ & & & & & & 0.019 \\
\hline No & 80 & $16(20 \%)$ & $74.7(63.1,86.2)$ & 1.00 (REF) & & \\
\hline Yes & 63 & $30(48 \%)$ & $46.5(30.6,62.4)$ & $2.08(1.13,3.85)$ & 0.019 & \\
\hline Age at diagnosis & 143 & $46(32 \%)$ & $59.8(48.9,70.8)$ & $1.06(1.03,1.08)$ & $<0.001$ & \\
\hline Last known age & 143 & $46(32 \%)$ & $59.8(48.9,70.8)$ & $1.031(1.009,1.054)$ & 0.006 & \\
\hline MACIS score & 143 & $46(32 \%)$ & $59.8(48.9,70.8)$ & $2.03(1.58,2.62)$ & $<0.001$ & $<0.001$ \\
\hline
\end{tabular}

Key: 'REF' stands for reference value (set at 1.00) in Cox univariate hazard ratio analysis.

\section{Pleural effusion characteristics}

Patients who developed a pleural effusion were further examined in which 70\% died during this 15-year study period. Half of the patients had exudative pleural effusions, and within this group, four out of the five had pleural fluid cytology that was positive for malignant cells (40\% were cytology positive). In the two patients who did not have an exudative pleural effusion or positive cytology, one died from the acute onset of a pleural effusion attributed to metastatic disease (Table 8). Patients with a pleural effusion survived a duration of $0.1-82.8$ months, and the patients who survived longer had RAIavid disease (Table 8).

\section{Discussion}

This study aimed to evaluate the impact of pleural effusion on OS and CSS in patients with FDTC and distant metastases and compare this novel risk factor to other well-known prognostic variables. This is the first study to investigate pleural effusion as a prognostic variable in metastatic follicular-cell-derived thyroid carcinoma, and with this information, providers can inform patients about their prognosis more effectively. Outside of lymph nodes, the lung is the most common site of distant metastases (12), and it is important for providers to be aware that if a patient develops a pleural effusion in the setting of lung metastases, this portends a worse prognosis than any other prognostic variable.

Prior studies have demonstrated that increased age (and age $\geq 45$ years), multisite metastases, osseous metastasis, incomplete tumor resection, and a higher MACIS score were associated with poor prognosis in patients with thyroid carcinoma $(2,3,4,8)$. In addition to these prognostic variables, distant metastases and increasing tumor size were identified as independent poor prognostic risk factors for follicular thyroid cancer $(5,8)$. Our data are consistent with these previous findings with similar OS and CSS hazard ratios compared to what was previously reported. Malignant pleural effusion has previously been

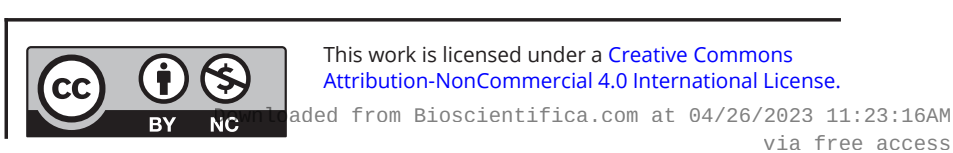


Table 5 Univariate cancer-specific survival summary.

\begin{tabular}{l}
\hline Variable \\
\hline Age > or $=45$ \\
No \\
Yes \\
Papillary cancer \\
Papillary thyroid cancer \\
Other thyroid cancer \\
Metastasis location \\
Lung only \\
Bone only \\
Other/multisite \\
Multiple metastatic locations \\
No \\
Yes \\
Completeness of surgery \\
Tumor completely resected \\
Tumor incompletely resected \\
Visible metastasis on lung \\
No \\
Yes \\
Visible metastasis on bone \\
No \\
Yes \\
Visible metastasis on other organs \\
No \\
Yes \\
Age at diagnosis \\
Last known age \\
MACIS score \\
\hline
\end{tabular}

\begin{tabular}{|c|c|c|}
\hline$n$ & Events & $\begin{array}{c}\text { 15-year survival } \\
\%(95 \% \mathrm{Cl})\end{array}$ \\
\hline 36 & $6(17 \%)$ & $89.6(78.2,100.0)$ \\
\hline 101 & $30(30 \%)$ & $56.5(41.8,71.2)$ \\
\hline 101 & $23(23 \%)$ & $73.6(63.3,84.0)$ \\
\hline 36 & $13(36 \%)$ & $49.6(25.2,74.0)$ \\
\hline 44 & $4(9 \%)$ & $85.1(70.9,99.3)$ \\
\hline 12 & $1(8 \%)$ & $90.9(73.9,100.0)$ \\
\hline 81 & $31(38 \%)$ & $57.4(44.0,70.8)$ \\
\hline 57 & $5(9 \%)$ & $85.7(73.1,98.2)$ \\
\hline 80 & $31(39 \%)$ & $57.1(43.7,70.5)$ \\
\hline 107 & $21(20 \%)$ & $74.6(64.2,85.0)$ \\
\hline 30 & $15(50 \%)$ & $45.4(23.4,67.5)$ \\
\hline 21 & $5(24 \%)$ & $79.3(61.1,97.6)$ \\
\hline 116 & $31(27 \%)$ & $65.4(53.8,77.1)$ \\
\hline 71 & $9(13 \%)$ & $81.8(70.2,93.4)$ \\
\hline 66 & $27(41 \%)$ & $51.9(36.0,67.9)$ \\
\hline 78 & $12(15 \%)$ & $78.3(66.9,89.6)$ \\
\hline 59 & $24(41 \%)$ & $54.9(38.4,71.4)$ \\
\hline 137 & $36(26 \%)$ & $66.9(56.2,77.5)$ \\
\hline 137 & $36(26 \%)$ & $66.9(56.2,77.5)$ \\
\hline 137 & $36(26 \%)$ & $66.9(56.2,77.5)$ \\
\hline
\end{tabular}

\begin{tabular}{l}
\hline $\begin{array}{c}\text { Cox univariate } \\
\text { hazard ratio }(95 \% \mathrm{Cl})\end{array}$ \\
\hline $1.00(\mathrm{REF})$ \\
$4.15(1.43,12.02)$ \\
$1.00(\mathrm{REF})$ \\
$1.83(0.91,3.65)$ \\
$1.00(\mathrm{REF})$ \\
$0.94(0.10,8.54)$ \\
$3.75(1.32,10.67)$ \\
$1.00(\mathrm{REF})$ \\
$3.90(1.51,10.08)$ \\
$1.00(\mathrm{REF})$ \\
$2.35(1.18,4.67)$ \\
$1.00(\mathrm{REF})$ \\
$1.01(0.39,2.65)$ \\
$1.00(\mathrm{REF})$ \\
$3.20(1.49,6.88)$ \\
$1.00(\mathrm{REF})$ \\
$2.27(1.12,4.60)$ \\
$1.06(1.03,1.08)$ \\
$1.030(1.005,1.055)$ \\
$1.98(1.49,2.63)$ \\
\end{tabular}

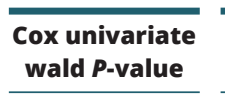

Cox univariate overall $P$-value

0.009

0.009

0.089

0.96

0.013

0.005

0.005

0.014

0.014

0.98

0.98

0.003

0.003

0.022

Key: 'REF' stands for reference value (set at 1.00) in Cox univariate hazard ratio analysis.

\begin{tabular}{|c|c|c|c|c|c|c|}
\hline Variable & $n$ & Events & $\begin{array}{c}\text { 15-year survival } \\
\%(95 \% \mathrm{Cl})\end{array}$ & $\begin{array}{c}\text { Cox univariate } \\
\text { hazard ratio }(95 \% \mathrm{Cl})\end{array}$ & $\begin{array}{l}\text { Cox univariate } \\
\text { wald } P \text {-value }\end{array}$ & $\begin{array}{l}\text { Cox univariate } \\
\text { overall } P \text {-value }\end{array}$ \\
\hline \multicolumn{7}{|l|}{ Overall survival: } \\
\hline Lung micronodules ( $\leq 9 \mathrm{~mm})$ & & & & & & 0.42 \\
\hline No & 8 & $3(38 \%)$ & $68.6(32.1,100.0)$ & 1.00 (REF) & & \\
\hline Yes & 45 & $13(29 \%)$ & $0.0(0.0,0.0)$ & $1.86(0.41,8.30)$ & 0.42 & \\
\hline Lung macronodules $(\geq 1 \mathrm{~cm})$ & & & & & & 0.056 \\
\hline No & 34 & $6(18 \%)$ & $0.0(0.0,0.0)$ & 1.00 (REF) & & \\
\hline Yes & 19 & $10(53 \%)$ & $39.9(13.2,66.6)$ & $2.74(0.97,7.70)$ & 0.056 & \\
\hline Pleural effusion & & & & & & 0.001 \\
\hline No & 43 & $9(21 \%)$ & $68.8(50.7,86.8)$ & 1.00 (REF) & & \\
\hline Yes & 10 & $7(70 \%)$ & $20.0(0.0,53.6)$ & $5.74(1.97,16.73)$ & 0.001 & \\
\hline \multicolumn{7}{|l|}{ Cancer-specific survival: } \\
\hline Lung micronodules ( $\leq 9 \mathrm{~mm}$ ) & & & & & & 0.47 \\
\hline No & 8 & $3(38 \%)$ & $68.6(32.1,100.0)$ & 1.00 (REF) & & \\
\hline Yes & 45 & $12(27 \%)$ & $0.0(0.0,0.0)$ & $1.75(0.39,7.89)$ & 0.47 & \\
\hline Lung macronodules $(\geq 1 \mathrm{~cm})$ & & & & & & 0.10 \\
\hline No & 34 & $6(18 \%)$ & $0.0(0.0,0.0)$ & 1.00 (REF) & & \\
\hline Yes & 19 & $9(47 \%)$ & $42.4(14.5,70.3)$ & $2.43(0.84,7.02)$ & 0.10 & \\
\hline Pleural effusion & & & & & & 0.006 \\
\hline No & 43 & $9(21 \%)$ & $68.8(50.7,86.8)$ & 1.00 (REF) & & \\
\hline Yes & 10 & $6(60 \%)$ & $22.9(0.0,60.8)$ & $4.93(1.59,15.26)$ & 0.006 & \\
\hline
\end{tabular}

Key: 'REF' stands for reference value (set at 1.00) in Cox univariate hazard ratio analysis.

https://ec.bioscientifica.com

https://doi.org/10.1530/EC-20-0193 (c) 2020 The authors Published by Bioscientifica Ltd

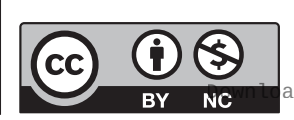

This work is licensed under a Creative Commons Attribution-NonCommercial 4.0 International License. ded from Bioscientifica.com at 04/26/2023 11:23:16AM via free access 


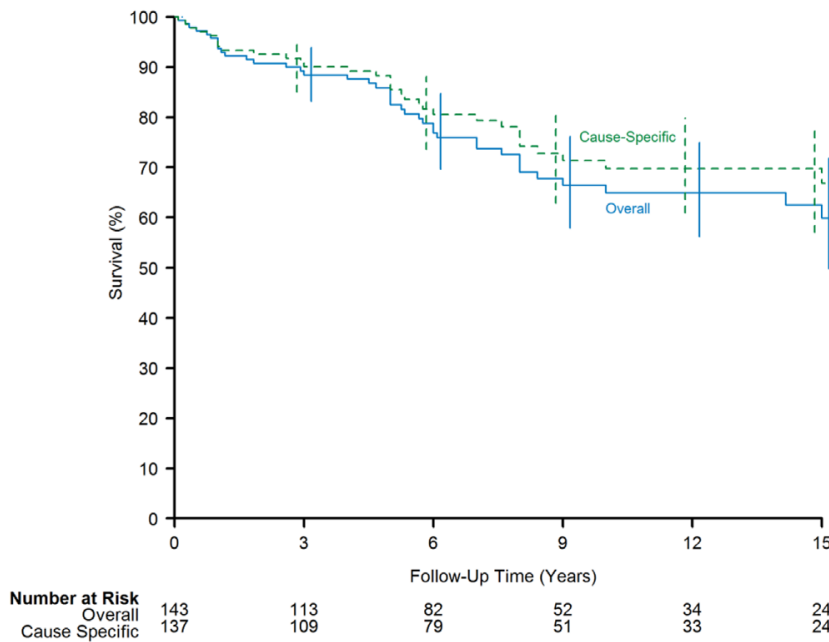

\section{Figure 1}

Kaplan-Meier curve for 15 -year overall- and cause-specific survival is shown among all patients.

demonstrated to be associated with poor outcomes in other types of malignancy $(12,13,14,15,16)$. It is noted that malignant pleural effusion complicates the clinical course in $0.6 \%$ of adult patients with papillary thyroid cancer and greatly shortens survival time in all cases (17, $18,19,20,21,22,23,24,25,26)$. Our data support these studies, and we further demonstrated that the hazard ratio was highest for patients with lung metastases who developed a pleural effusion, when compared to other prognostic variables. In our study, lung metastases with development of pleural effusion had a hazard ratio higher than the second worst prognostic variable's hazard ratio (age $\geq 45$ years). With the significance of pleural effusion having a higher hazard ratio than other variables, details of these patients were further sought.

When examining our data closely, $70 \%$ of all cases that developed a pleural effusion died within 15 years, and the survival after development of pleural effusion was from 0.1 to 82.8 months with a median survival of 15 months. This is similar to prior studies that found pleural effusion preceded death by a median of 10-11 months $(17,18)$. Specifically, most patients were dying from respiratory failure or a complication of fluid accumulation (e.g. pneumonia). What is surprising is that two-thirds of the patients who survived had cytology-proven malignant pleural effusions (with the remaining case not having their fluid sampled). This may be that, in these scenarios, the patients underwent multiple interventions (thoracentesis, pleurX drains, thoracotomy) or more aggressive therapeutic interventions (tyrosine kinase inhibitors, multikinase inhibitors).

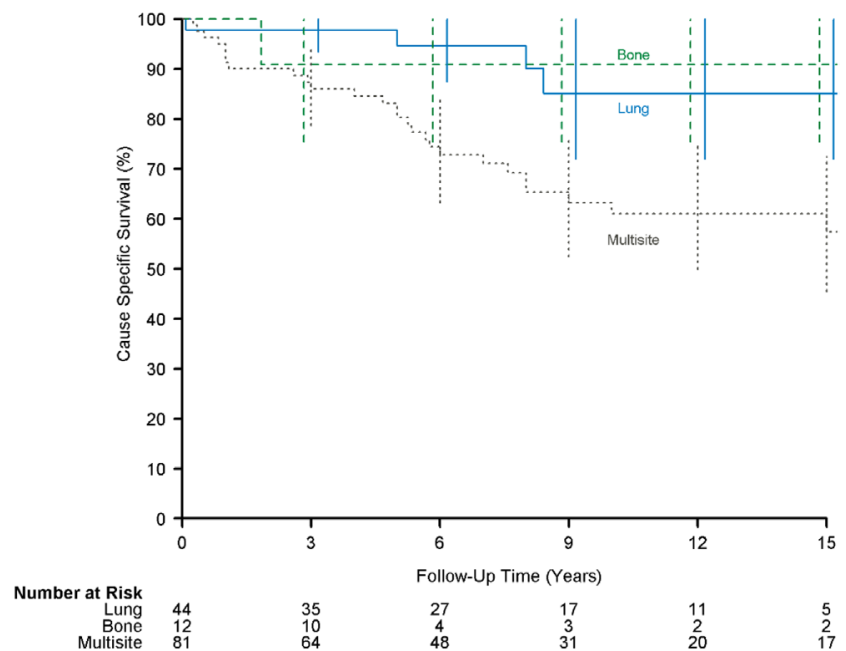

Figure 2

Kaplan-Meier curve for 15-year cause-specific survival by site is shown among all patients.

In 2014 and 2015, it was reported that the tyrosine kinase inhibitor sorafenib and the multikinase inhibitor lenvatinib increase progression-free survival in patients with RAI-refractory metastatic thyroid carcinoma $(27,28)$. Since that time, there has been a case report demonstrating a notable decrease in a malignant pleural effusion after treatment with sorafenib in RAI-refractory follicular thyroid carcinoma (29) and a subsequent report of rapid pleural effusion accumulation after discontinuation of lenvatinib (30). This is important to note, as it seems that currently available therapeutic options have the potential to improve the prognostic outcomes of patients who have metastatic thyroid carcinoma, that is, radioiodine

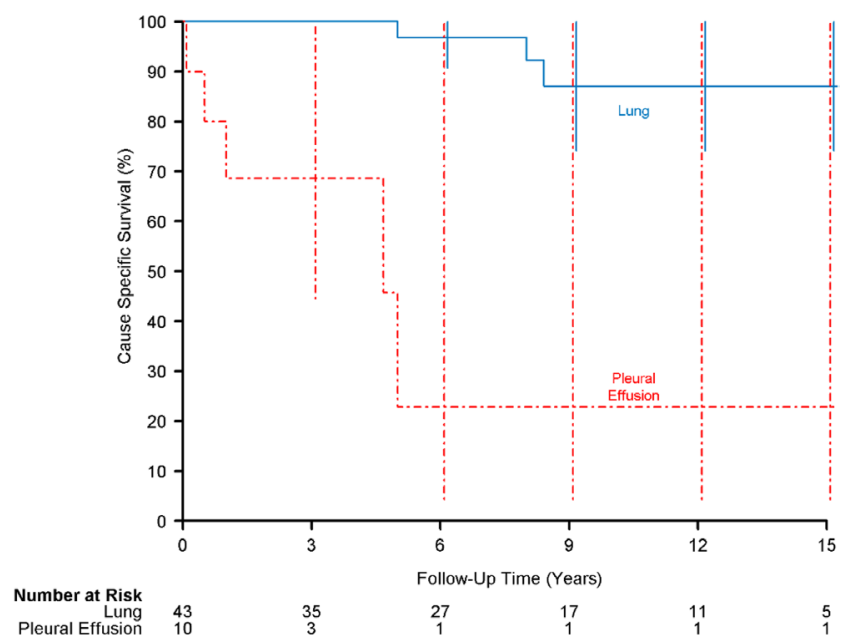

Figure 3

Kaplan-Meier curve for 15-year cause-specific survival in patients with lung metastases with and without pleural effusion.

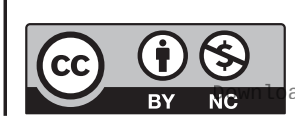

This work is licensed under a Creative Commons Attribution-NonCommercial 4.0 International License. ded from Bioscientifica.com at 04/26/2023 11:23:16AM via free access 
Table 7 Multivariable overall survival and cancer-specific survival analyses.

\begin{tabular}{|c|c|c|c|c|c|c|}
\hline Variable & $n$ & Events & $\begin{array}{l}\text { 15-year survival } \\
\%(95 \% \mathrm{Cl})\end{array}$ & $\begin{array}{c}\text { Cox multivariable } \\
\text { hazard ratio }(95 \% \mathrm{Cl})\end{array}$ & $\begin{array}{c}\text { Cox univariate } \\
\text { wald P-value } \\
(n=143)\end{array}$ & $\begin{array}{l}\text { Cox univariate } \\
\text { overall P-value } \\
(n=143)\end{array}$ \\
\hline \multicolumn{7}{|l|}{ Overall survival analysis: } \\
\hline $\begin{array}{l}\text { Lung metastasis } \\
\text { at presentation }\end{array}$ & & & & & & 0.002 \\
\hline No & 90 & $30(33 \%)$ & $62.3(49.7,74.8)$ & 1.00 (REF) & & \\
\hline $\begin{array}{l}\text { Yes, without pleural } \\
\text { effusion }\end{array}$ & 43 & $9(21 \%)$ & $68.8(50.7,86.8)$ & $0.60(0.26,1.41)$ & 0.24 & \\
\hline Yes, with pleural effusion & 10 & $7(70 \%)$ & $20.0(0.0,53.6)$ & $4.42(1.68,11.62)$ & 0.003 & \\
\hline $\begin{array}{l}\text { Undetectable thyroglobulin } \\
\text { post RAI treatment }\end{array}$ & & & & & & 0.049 \\
\hline No & 115 & $36(31 \%)$ & $62.7(51.1,74.4)$ & 1.00 (REF) & & \\
\hline Yes & 28 & $10(36 \%)$ & $45.8(15.6,76.0)$ & $2.168(1.003,4.686)$ & 0.049 & \\
\hline RAl treatment & & & & & & $<0.001$ \\
\hline No & 12 & $7(58 \%)$ & $0.0(0.0,0.0)$ & 1.00 (REF) & & \\
\hline Yes & 131 & $39(30 \%)$ & $62.7(51.4,74.1)$ & $0.18(0.07,0.47)$ & $<0.001$ & \\
\hline MACIS score & 143 & $46(32 \%)$ & $59.8(48.9,70.8)$ & $1.96(1.51,2.55)$ & $<0.001$ & $<0.001$ \\
\hline \multicolumn{7}{|l|}{$\begin{array}{l}\text { Cancer-specific survival } \\
\text { analysis: }\end{array}$} \\
\hline RAl treatment & & & & & & 0.005 \\
\hline No & 12 & $6(50 \%)$ & $0.0(0.0,0.0)$ & 1.00 (REF) & & \\
\hline Yes & 125 & $30(24 \%)$ & $69.9(59.1,80.7)$ & $0.23(0.09,0.65)$ & 0.005 & \\
\hline $\begin{array}{l}\text { Lung metastasis at } \\
\text { presentation }\end{array}$ & & & & & & 0.008 \\
\hline No & 84 & $21(25 \%)$ & $71.2(59.0,83.4)$ & 1.00 (REF) & & \\
\hline $\begin{array}{l}\text { Yes, without pleural } \\
\text { effusion }\end{array}$ & 43 & $9(21 \%)$ & $68.8(50.7,86.8)$ & $1.05(0.44,2.50)$ & 0.92 & \\
\hline Yes, with pleural effusion & 10 & $6(60 \%)$ & $22.9(0.0,60.8)$ & $5.21(1.79,15.12)$ & 0.002 & \\
\hline MACIS score & 137 & $36(26 \%)$ & $66.9(56.2,77.5)$ & $1.86(1.38,2.49)$ & $<0.001$ & $<0.001$ \\
\hline
\end{tabular}

Key: 'REF' stands for reference value (set at 1.00) in Cox multivariable hazard ratio analysis.

refractory, in addition to help resolve and prevent the re-accumulation of pleural effusion in these patients. This, in addition to addressing limitations from this study, may be an important future area of study.

One limitation of this study is that it is a retrospective study and there are inherent limitations in data that are available to be collected. Specifically, some patients did not have complete data, such as underlying thyroid disease upon presentation, and also the pathological subtype was not always present or obtainable for our pathologists to review. This latter concern is a considerable limitation in our study, as in these circumstances, we had to rely on interpretation from the referring outside institution for their pathological interpretation and did not have access to the histologic subtype. A second limitation of this study was that this study did not utilize socioeconomic status, access to care, or the Eastern Cooperative Oncology Group (ECOG) performance status as variables. This may have affected our results as patients with low socioeconomic status, limited access to care, and a low ECOG performance status would likely contribute to a decreased OS and CSS. A third limitation of this study is that the IRB was written

$\begin{array}{lr}\text { https://ec.bioscientifica.com } & \text { (c) } 2020 \text { The authors } \\ \text { https://doi.org/10.1530/EC-20-0193 } & \text { Published by Bioscientifica Ltd }\end{array}$

prior to the publication of the AJCC eighth edition for staging of malignancy, and therefore, the AJCC seventh edition staging system was used. This is unlikely to have an effect on our results, as our database included patients who were diagnosed or had presented between the years 1990 and 2010, and it is recommended that all newly diagnosed cases through December 31, 2017 continue to be staged by tumor registries according to the seventh edition staging system (31). A fourth limitation is that comparisons involving patients with pleural effusion had limited power and may be subject to influence by individual patient response. Therefore, these findings may be further validated with external corroboration and future studies with a larger cohort to ensure these findings are reliable.

\section{Conclusion}

Utilizing a 15-year retrospective chart review of patients presenting with FDTC and distant metastases, our study sought to identify and quantify the importance of novel

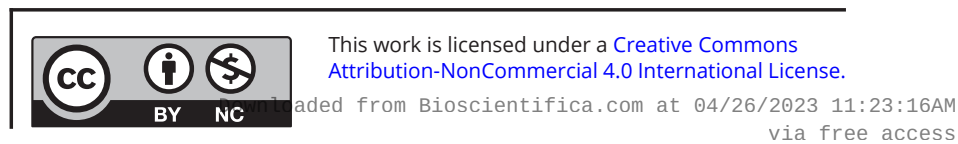



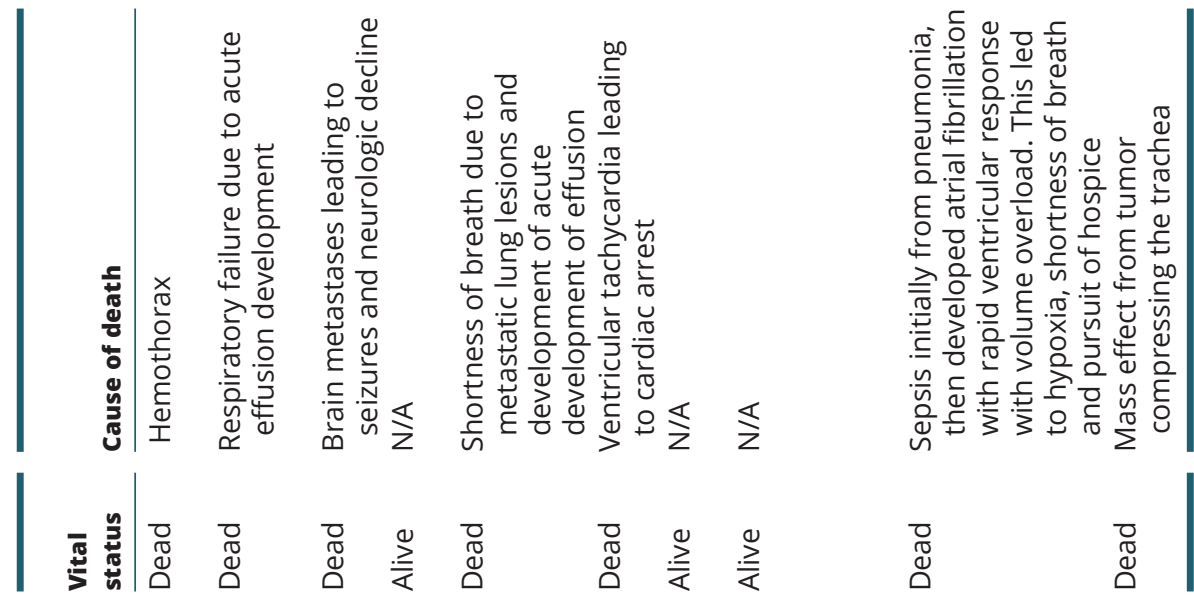

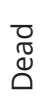
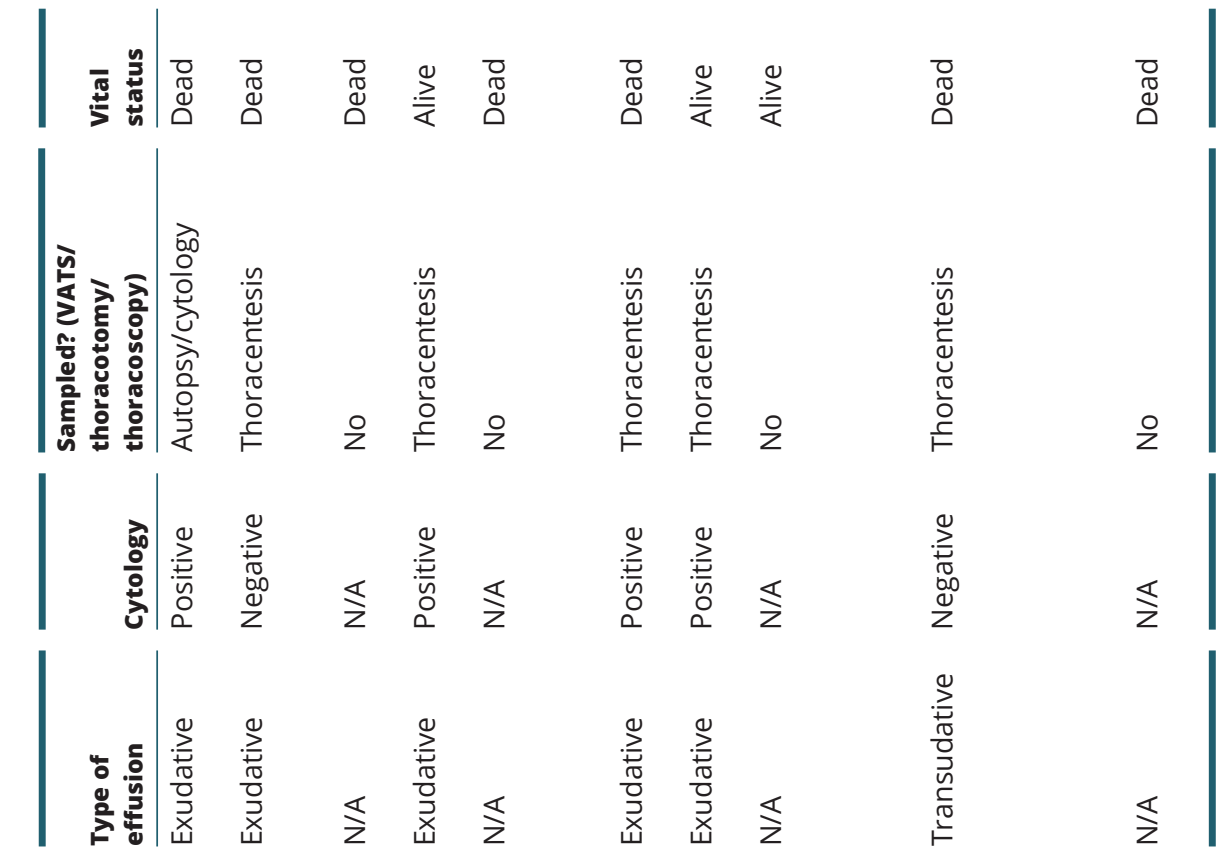

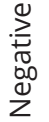
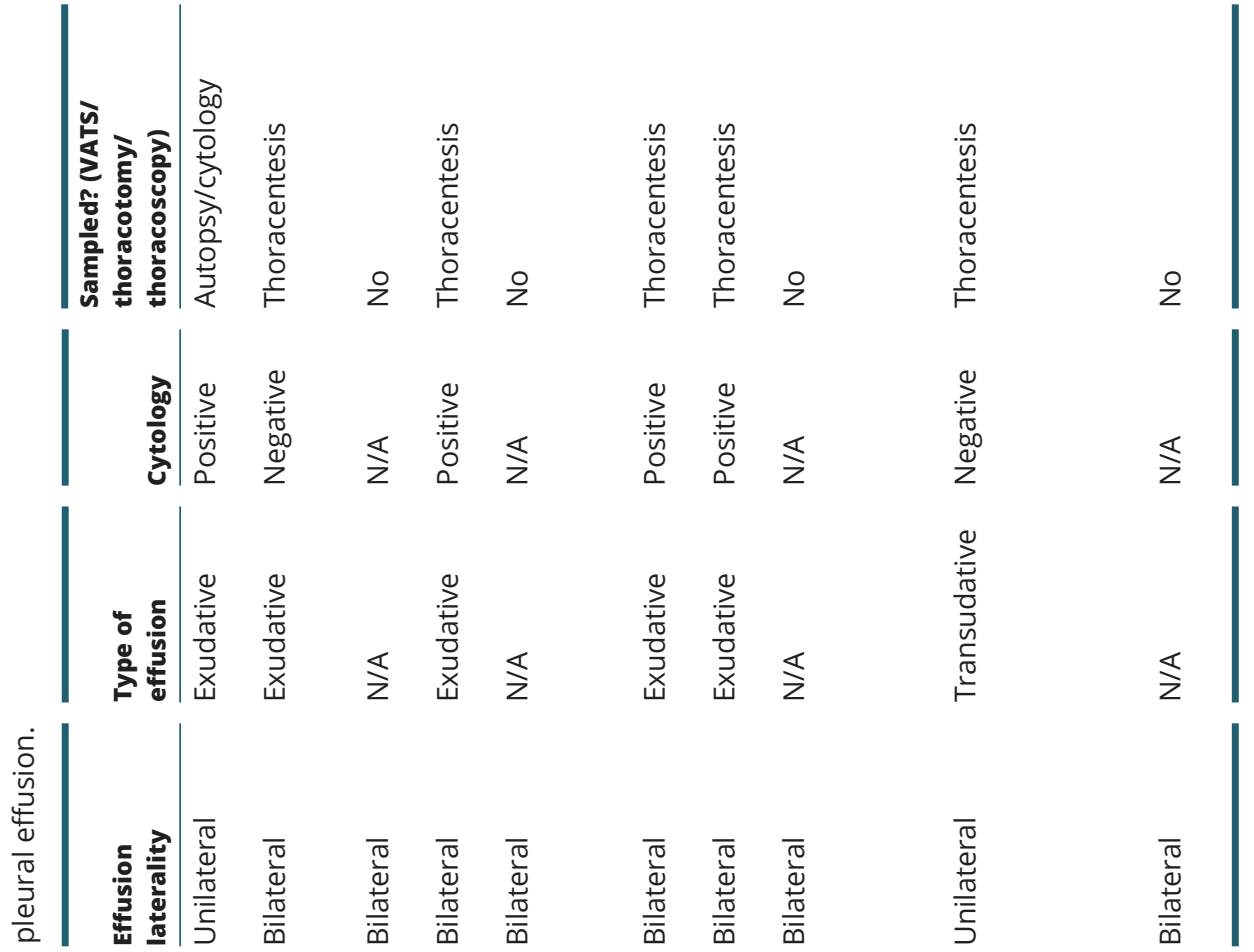

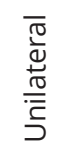
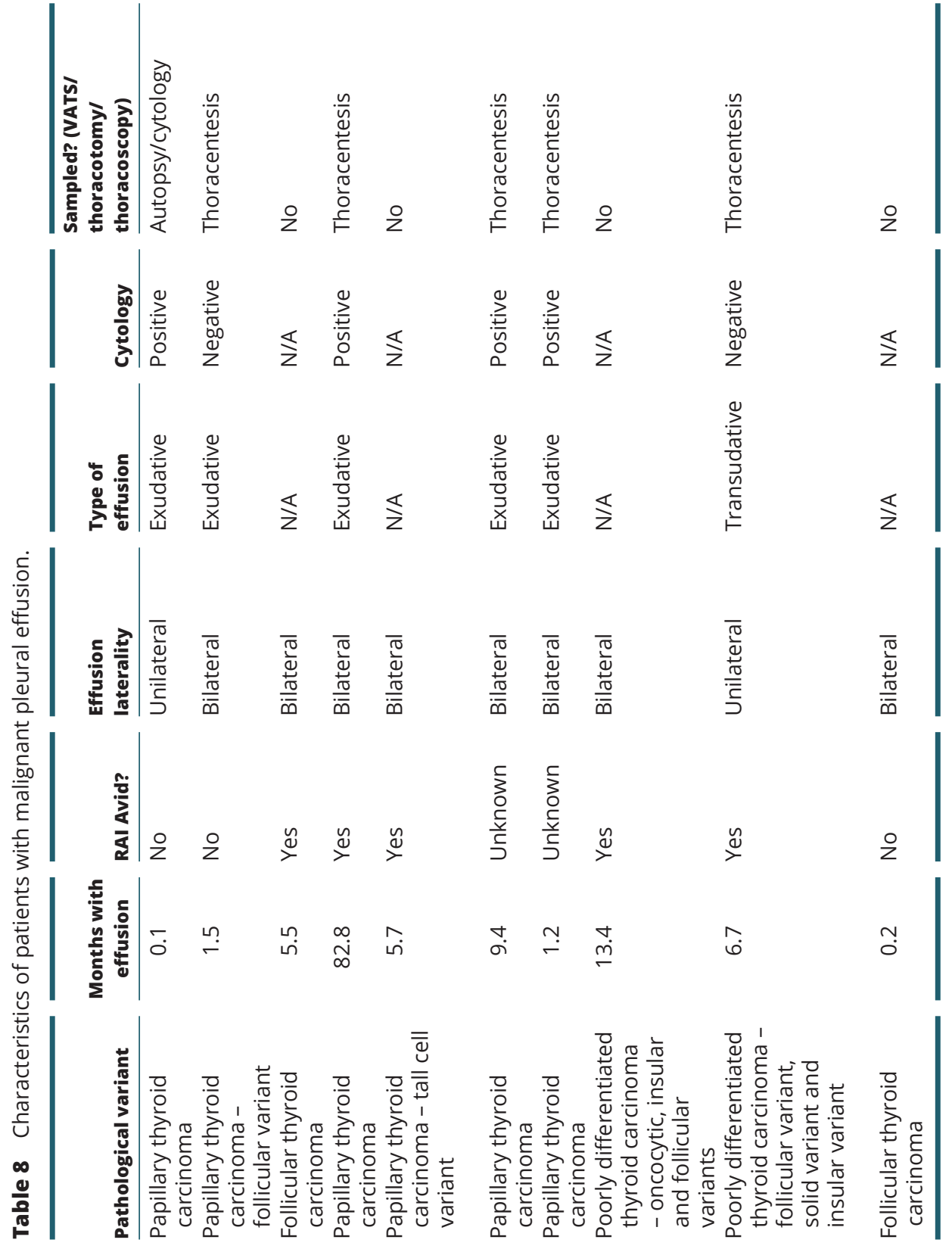

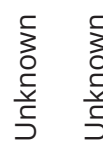

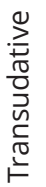

$\widehat{\vartheta}$

ֻุ
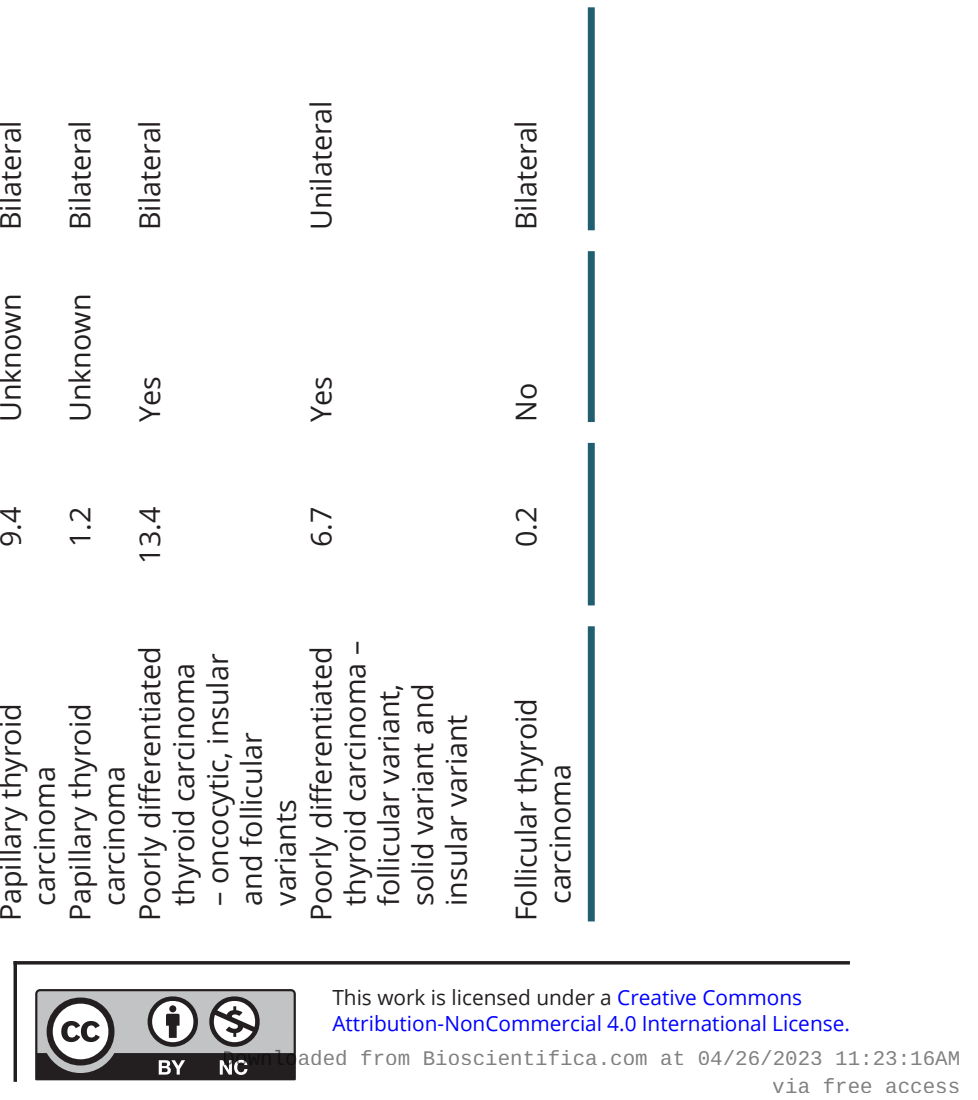
prognostic variables on OS and CSS. In this study, pleural effusion, in addition to age $\geq 45$ years, multisite metastases, incomplete tumor resection, visible metastases in bone, age at diagnosis, and a higher MACIS score were identified as significant risk factors for decreased overall- and cancerspecific survival. Pleural effusion is a novel prognostic finding and is associated with a more significant decrease in OS and CSS compared to other known prognostic variables in patients with FDTC with distant metastases.

\section{Supplementary materials}

This is linked to the online version of the paper at https://doi.org/10.1530/ EC-20-0193.

\section{Declaration of interest}

$\mathrm{C} \mathrm{N}$ has no direct conflict of interest. $\mathrm{C} \mathrm{N}$ is a member on the advisory committee or review panels for Exelixis and Nevro Corporation. The other authors have nothing to disclose.

\section{Funding}

This research did not receive any specific grant from any funding agency in the public, commercial or not-for-profit sector.

\section{Author contribution statement}

D T B contributed to data collection and wrote the manuscript. G B G designed the study and edited the manuscript. E F edited the manuscript. J F B performed the statistical analyses. C N designed the study and edited the manuscript.

\section{Acknowledgements}

D T B was the recipient of the AACE Domestic Resident Travel Grant and presented this at the 27th Annual Meeting \& Clinical Congress of American Association of Clinical Endocrinologists (AACE) in 2018.

\section{References}

1 National Cancer Institute. Surveillance, Epidemiology, and End Results Program. SEER stat fact sheets: thyroid cancer. Bethesda, MD, USA: National Cancer Institute. (available at: https://seer.cancer.gov/ statfacts/html/thyro.html)

2 Clive AO, Kahan BC, Hooper CE, Bhatnagar R, Morley AJ, ZahanEvans N, Bintcliffe OJ, Boshuizen RC, Fysh ET, Tobin CL, et al. Predicting survival in malignant pleural effusion: development and validation of the LENT prognostic score. Thorax 201469 1098-1104. (https://doi.org/10.1136/thoraxjnl-2014-205285)

3 The American Thoracic Society. Management of malignant pleural effusions. American Journal of Respiratory and Critical Care Medicine 2000162 1987-2001. (https://doi.org/10.1164/ajrccm.162.5.ats8-00)

4 Office of National Statistics. Cancer Statistics Registrations, England (Series MB1). Newport, UK: Office of National Statistics, Stationary Office, 2010.

5 Lin JD, Huang MJ, Juang JH, Chao TC, Huang BY, Chen KW, Chen JY, Li KL, Chen JF \& Ho YS. Factors related to the survival of papillary and follicular thyroid carcinoma patients with distant metastases. Thyroid 19999 1227-1235. (https://doi.org/10.1089/thy.1999.9.1227)
6 Lang BH, Wong KP, Cheung CY, Yat Wan KY \& Lo CY. Evaluating the prognostic factors associated with cancer-specific survival of differentiated thyroid carcinoma presenting with distant metastasis. Annals of Surgical Oncology 201320 1329-1335. (https://doi. org/10.1245/s10434-012-2711-x)

7 Samaan NA, Schultz PN, Hickey RC, Goepfert H, Haynie TP, Johnston DA \& Ordonez NG. The results of various modalities of treatment of well differentiated thyroid carcinomas: a retrospective review of 1599 patients. Journal of Clinical Endocrinology and Metabolism 199275 714-720. (https://doi.org/10.1210/ jcem.75.3.1517360)

8 Passler C, Scheuba C, Prager G, Kaczirek K, Kasere K, Zettinig G \& Niederle B. Prognostic factors of papillary and follicular thyroid cancer: differences in an iodine-replete endemic goiter region. Endocrine-Related Cancer 200411 131-139. (https://doi.org/10.1677/ erc.0.0110131)

9 Hay ID, Bergstralh EJ, Goellner JR, Ebersold JR \& Grant CS. Predicting outcome in papillary thyroid carcinoma: development of a reliable prognostic scoring system in a cohort of 1779 patients surgically treated at one institution during 1940 through 1989. Surgery 1993 114 1050-1057; discussion 1057-1058

10 Edge SB, Byrd DR, Compton CC, Fritz AG, Greene FL \& Trotti A (eds). AJCC Cancer Staging Manual, 7th ed. New York, NY, USA: SpringerVerlag, 2010.

11 Haugen BR, Alexander EK, Bible KC, Doherty GM, Mandel SJ, Nikiforov YE, Pacini F, Randolph GW, Sawka AM, Schlumberger M, et al. 2015 American Thyroid Association management guidelines for adult patients with thyroid nodules and differentiated thyroid cancer: the American Thyroid Association guidelines task force on thyroid nodules and differentiated thyroid cancer. Thyroid 201626 1-133. (https://doi.org/10.1089/thy.2015.0020)

12 Wang LY, Palmer FL, Nixon IJ, Thomas D, Patel SG, Shaha AR, Shah JP, Tuttle RM \& Ganly I. Multi-organ distant metastases confer worse disease-specific survival in differentiated thyroid cancer. Thyroid 201424 1594-1599. (https://doi.org/10.1089/thy.2014.0173)

13 Suguira S, Ando Y, Minami H, Ando M, Sakai S \& Shimokata K. Prognostic value of pleural effusion in patients with non-small cell lung cancer. Clinical Cancer Research 19973 47-50.

14 Jett JR, Scott WJ, Rivera MP, Sause WT \& American College of Chest Physicians. Guidelines on treatment of stage IIIB non-small cell lung cancer. Chest 2003123 (Supplement) 221S-225S. (https://doi. org/10.1378/chest.123.1_suppl.221s)

15 Goldstraw P, Crowley J, Chansky K, Giroux DJ, Groome PA, RamiPorta R, Postmus PE, Rusch V, Sobin L, International Association for the Study of Lung Cancer International Staging Committee, et al. The IASLC Lung Cancer Staging Project: proposals for the revision of the TNM stage groupings in the forthcoming (seventh) edition of the TNM classification of malignant tumours. Journal of Thoracic Oncology 20072 706-714. (https://doi.org/10.1097/ JTO.0b013e31812f3c1a)

16 Morgensztern D, Wagar S, Subramanian J, Trinkaus K \& Govindan R. Prognostic impact of malignant pleural effusion at presentation in patients with metastatic non-small-cell lung cancer. Journal of Thoracic Oncology 20127 1485-1489. (https://doi.org/10.1097/ JTO.0b013e318267223a)

17 Vassipolou-Sellin R \& Sneige N. Pleural effusion in patients with differentiated papillary thyroid cancer. Southern Medical Journal 1994 87 1111-1116. (https://doi.org/10.1097/00007611-19941100000010)

18 Tamura T, Shiozawa T, Satoh H, Kurishima K, Kagohashi K, Takayashiki N \& Hizawa N. Pleural fluid due to papillary thyroid cancer. Oncology Letters 201918 962-966. (https://doi.org/10.3892/ ol.2019.10383)

19 Yavarovich E \& Lamb C. Metastatic thyroid carcinoma manifesting as left sided pleural effusion. Chest 2019156 (Supplement) A1281. (https://doi.org/10.1016/j.chest.2019.08.1152) https://ec.bioscientifica.com https://doi.org/10.1530/EC-20-0193 (c) 2020 The authors Published by Bioscientifica Lto
This work is licensed under a Creative Commons Attribution-NonCommercial 4.0 International License. ded from Bioscientifica.com at 04/26/2023 11:23:16AM 
20 Vernon AN, Sheeler LR, Biscotti CV \& Stoller JK. Pleural effusion resulting from metastatic papillary carcinoma of the thyroid. Chest 1992101 1448-1450. (https://doi.org/10.1378/chest.101.5.1448)

21 Tomoda C, Ogimi Y, Saito F, Masaki C, Akaishi J, Matsuzu K, Suzuki A, Uruno T, Ohkuwa K, Shibuya H, et al. Outcome and characteristics of patients with malignant pleural effusion from differentiated thyroid carcinoma. Endocrine Journal 201663 257-261. (https://doi.org/10.1507/endocrj.EJ15-0631)

22 Kosmas K, Tsonou A, Mitropoulou G, Salemi E, Kazi D \& Theofanopoulou A. Malignant pleural effusion from papillary thyroid carcinoma diagnosed by pleural effusion cytology: a case report. Diagnostic Cytopathology 201846 204-207. (https://doi. org/10.1002/dc.23824)

23 Vyas M \& Harigopal M. Metastatic thyroid carcinoma presenting as malignant pleural effusion: a cytologic review of 5 cases. Diagnostic Cytopathology 201644 1085-1089. (https://doi.org/10.1002/dc.23547)

24 Sakamoto RI, Sumida LC, Lum CA \& Tauchi-Nishi PS. Recurrent papillary thyroid carcinoma with pleural metastasis diagnosed by effusion cytology: a report of cases with clinicopathologic correlation. Hawai'i Journal of Medicine and Public Health 201574 51-56.

25 Lew M, Pang JC, Roh MH \& Jing X. Cytologic features and immunocytochemical profiles of malignant effusions with metastatic papillary thyroid carcinoma: a case series from a single institution. Acta Cytologica 201559 412-417. (https://doi. org/10.1159/000441647)

26 Olson MT, Nuransoy A \& Ali SZ. Malignant pleural effusion resulting from metastasis of thyroid primaries: a cytomorphological analysis. Acta Cytologica 201357 177-183. (https://doi org/10.1159/000345696)

27 Brose MS, Nutting CM, Jarzab B, Elisei R, Siena S, Basthold L, de la Fouchardiere C, Pacini F, Paschke R, KeeShong Y, et al. Sorafenib in locally advanced or metastatic, radioactive iodine-refractory, differentiated thyroid cancer: a randomized, double-blind, phase 3 trial. Lancet 2014384 319-328.

28 Schlumberger M, Tahara M, Wirth LJ, Robinson B, Brose MS, Elisei R, Habra MA, Newbold K, Shah MH, Hoff AO, et al. Lenvatinib versus placebo in radioiodine-refractory thyroid cancer. New England Journal of Medicine 2015372 621-630. (https://doi.org/10.1056/ NEJMoa1406470)

29 Liu M, Shen Y, Ruan M, Li M \& Chen L. Notable decrease of malignant pleural effusion after treatment with sorafenib in radioiodine-refractory follicular thyroid carcinoma. Thyroid $20142 \mathbf{4}$ 1179-1183. (https://doi.org/10.1089/thy.2013.0703)

30 Uchida T, Yamaguchi H, Nagamine K, Yonekawa T, Nakamura E, Shibata N, Kawano F, Asada Y \& Nakazato M. Rapid pleural effusion after discontinuation of lenvatinib in a patient with pleural metastasis from thyroid cancer. Endocrinology, Diabetes and Metabolism Case Reports 20192019 EDM180158. (https://doi. org/10.1530/EDM-18-0158)

31 Tuttle RM, Haugen B \& Perrier ND. Updated American Joint Committee on Cancer/tumor-node-metastasis staging system for differentiated and anaplastic thyroid cancer (eighth edition): what changed and why? Thyroid 201727 751-756. (https://doi. org/10.1089/thy.2017.0102)

Received in final form 12 June 2020

Accepted 28 July 2020

Accepted Manuscript published online 28 July 2020 https://ec.bioscientifica.com

https://doi.org/10.1530/EC-20-0193 (c) 2020 The authors Published by Bioscientifica Ltd
This work is licensed under a Creative Commons Attribution-NonCommercial 4.0 International License. ded from Bioscientifica.com at 04/26/2023 11:23:16AM 